\title{
Chalcogenide $\mathrm{As}_{2} \mathrm{~S}_{3}$ Sidewall Bragg Gratings Integrated on $\mathrm{LiNbO}_{3}$ Substrate*
}

\author{
Xin Wang ${ }^{1}$, Aiting Jiang ${ }^{2}$, Christi Madsen ${ }^{1}$ \\ ${ }^{1}$ Department of Electrical and Computer Engineering, Texas A\&M University, College Station, USA \\ ${ }^{2}$ Department of Electrical and Computer Engineering, University of Texas at Austin, Austin, USA \\ Email: xinwang@neo.tamu.edu
}

Received January 9, 2013; revised February 10, 2013; accepted February 17, 2013

\begin{abstract}
This paper introduces the design and applications of integrated $\mathrm{As}_{2} \mathrm{~S}_{3}$ sidewall Bragg gratings on $\mathrm{LiNbO}_{3}$ substrate. The grating reflectance and bandwidth are analyzed with coupled-mode theory. Coupling coefficients are computed by taking overlap integration. Numerical results for uniform gratings, phase-shifted gratings and grating cavities as well as electro-optic tunable gratings are presented. These integrated $\mathrm{As}_{2} \mathrm{~S}_{3}$ sidewall gratings on $\mathrm{LiNbO}_{3}$ substrate provide an approach to the design of a wide range of integrated optical devices including switches, laser cavities, modulators, sensors and tunable filters.
\end{abstract}

Keywords: Waveguides; Bragg Gratings; Coupled-Mode Theory

\section{Introduction}

Bragg gratings have been widely used in integrated optical devices such as switches, filters, laser, modulators and wavelength division multiplexing [1-5]. Recently, sidewall (or lateral) Bragg gratings show obvious advantages over surface or volume Bragg gratings, owing to its low insertion loss, compact size and relaxed fabrication tolerance [6-14]. On the one hand, lithium niobate $\left(\mathrm{LiNbO}_{3}\right)$ has become an attractive material for integrated optical applications because of its outstanding electro-optical, acousto-optical and optical transmission properties [15]. In $\mathrm{LiNbO}_{3}$ substrate, high-quality channel waveguide can be made by annealed proton exchange (APE) [16] or by thin film titanium (Ti) diffusion [17]. The mode size of such channel waveguides is comparable with optical fiber, making their coupling loss extremely small. On the other hand, arsenic tri-sulfide $\left(\mathrm{As}_{2} \mathrm{~S}_{3}\right)$ is one type of amorphous chalcogenide glass that can be fabricated into low-loss waveguide structures using conventional silicon lithography techniques for both near-IR and mid-IR applications $[18,19] . \mathrm{As}_{2} \mathrm{~S}_{3}$ also possesses a large refractive index contrast that enables strong optical confinement in narrow waveguides with wide bend radii [20]. Besides, the optical mode can be vertically coupled from the $\mathrm{LiNbO}_{3}$ channel waveguide into the $\mathrm{As}_{2} \mathrm{~S}_{3}$ waveguide through tapering structures [21]. Therefore, the combination of $\mathrm{As}_{2} \mathrm{~S}_{3}$ waveguide structure and $\mathrm{LiNbO}_{3}$

"This work is funded by National Science Foundation (NSF) under grant EEC-0540832. substrate provides a powerful hybrid integrated optical platform for many applications [17,20-22].

In this paper, we explore the spectral properties of integrated $\mathrm{As}_{2} \mathrm{~S}_{3}$ sidewall gratings on $\mathrm{LiNbO}_{3}$ substrate and investigate their device applications in integrated optics. Sidewall gratings of the sinusoidal, trapezoidal and square shapes are analyzed with coupled-mode theory [23-25]. Numerical results from uniform sidewall gratings under weak coupling and strong coupling conditions are compared with coupled-mode theory. In simulations, material dispersions are considered by applying Sellmeier equations $[26,27]$. By introducing multiple phase shifting spacers into uniform sidewall gratings, multi-channel transmission filter was implemented. Besides, electro-optical tunable transmission filter is designed utilizing the unique electro-optical property of $\mathrm{LiNbO}_{3}$ substrate. A tuning rate of $\sim 4 \mathrm{pm} / \mathrm{V}$ was predicted from a narrowband transmission filter based on single phase-shifted sidewall Bragg gratings. Ultrahigh Q-factor of over $10^{6}$ is also proposed by adjusting the resonant cavity length formed by two identical uniform gratings. This type of integrated $\mathrm{As}_{2} \mathrm{~S}_{3}$ sidewall Bragg gratings on lithium niobate substrate provide an approach to the design of a wide range of integrated optical devices such as optical switches, laser cavities, modulators, sensors and tunable filters.

\section{Theory}

\subsection{Coupled-Mode Equations}

General principle of the mode coupling resulting from 
periodic dielectric perturbation was rigorously derived in Ref. [23,24]. For Bragg grating reflectors, only two contra-directional waves are involved. For $y$-propagation, the coupled-mode equations are given by

$$
\begin{aligned}
& \frac{\mathrm{d} A_{1}(y)}{\mathrm{d} y}=-j k A_{2}(y) \mathrm{e}^{j \Delta \beta y} \\
& \frac{\mathrm{d} A_{2}(y)}{\mathrm{d} y}=j k^{*} A_{1}(y) \mathrm{e}^{-j \Delta \beta y}
\end{aligned}
$$

where $\Delta \beta=\beta_{1}-\beta_{2}-K, K=m \frac{2 \pi}{\Lambda}$.

$A_{1}$ and $A_{2}$ are complex amplitudes of the normalized waves with propagation constants of $\beta_{1}$ and $\beta_{2}$, respectively. $\Delta \beta$ is phase mismatch; $\Lambda$ is grating period; $K$ is grating wavenumber and $\mathrm{m}$ is a positive integer indicating grating order; $k$ is the coupling coefficient. The power transfer between the two waves through the grating with a length of $L$ is derived as

$$
R=\left|\frac{A_{2}(0)}{A_{1}(0)}\right|^{2}=\frac{|k|^{2} \sinh ^{2} s L}{s^{2} \cosh ^{2} s L+(\Delta \beta / 2)^{2} \sinh ^{2} s L}
$$

where $s^{2}=k^{*} k-(\Delta \beta / 2)^{2}$.

The Bragg grating reflectance as a function of phase mismatch $\Delta \beta L / 2$ at different coupling strengths for a 1 $\mathrm{cm}$-long Bragg grating is shown in Figure 1. The grating reflectance decreases smoothly as $\Delta \beta / 2$ increases from 0 to $k$. When $\Delta \beta$ is beyond $k$, the value of $\mathrm{s}$ becomes imaginary and the waves change from exponential to sinusoidal. It also shows that the grating reflectance increases as $k L$ increases under phase match condition $\Delta \beta$ $=0$, in which case, the power transfer reaches its maximum value at $R_{\max }=\tanh ^{2}(k L)$. The corresponding grating period is determined by $\Lambda=m \lambda_{0} /\left(2 n_{\text {eff }}\right)$ where $\lambda_{0}$ is Bragg wavelength and $n_{\text {eff }}$ is the mode effective index of the forward propagating wave $\beta_{1}$.

The reflection bandwidth is defined as the wavelength span between the first two minima in reflection spectrum

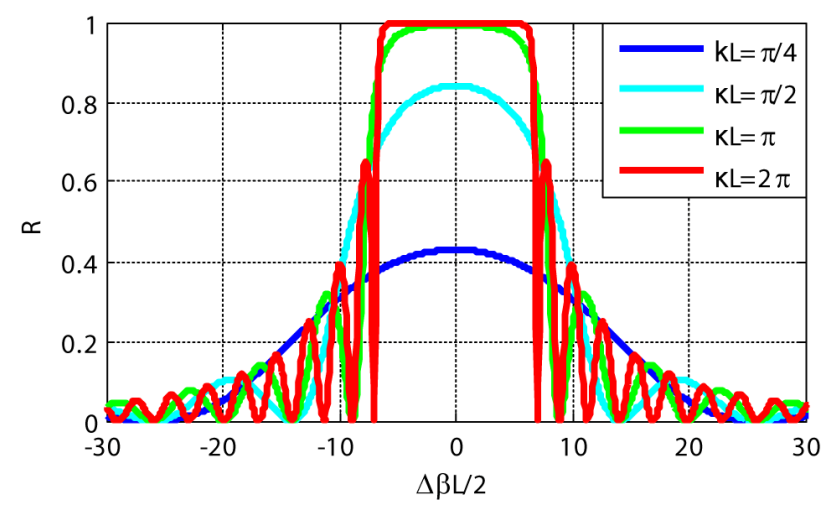

Figure 1. Bragg grating reflectance for different phase mismatches. as described by Equation (4). The bandwidth $\Delta \lambda$ is determined by the grating length $\mathrm{L}$ under weak coupling condition $k L \ll \pi$. For strong coupling condition $k L \gg$ $\pi, \Delta \lambda$ is directly proportional to coupling coefficient $k$.

$$
\Delta \lambda=\frac{\lambda_{0}^{2}}{2 \pi n_{\mathrm{eff}} L} \sqrt{(k L)^{2}+\pi^{2}}
$$

\subsection{Fourier Coefficients}

As depicted in Figure 2, the sidewall Bragg gratings are engraved in both sidewalls of $\mathrm{As}_{2} \mathrm{~S}_{3}$ strips on $\mathrm{LiNbO}_{3}$. The grating period is $\Lambda$. Duty cycle (DC) is defined as the fractional width with high index material in one grating period and thus $\mathrm{DC}=\tau / \Lambda$ where $\tau$ is the width of high index material in one period. The rise/fall width is denoted by $\tau_{r} . \Delta W$ is the grating depth which is a measure of the index perturbation strength. $W$ and $t$ are the width and thickness of equivalent unperturbed $\mathrm{As}_{2} \mathrm{~S}_{3}$ strip waveguide, respectively.

Arbitrary grating shape along $x$ - and $z$-axis with periodic perturbation of index modulation along $y$-axis can be expressed in the form of Taylor expansion, as described by

$$
\begin{aligned}
& \Delta \varepsilon(x, y)=u(x) \Delta \varepsilon(y) \\
& u(x)=1,(0<x<t) \\
& \begin{array}{c}
\Delta \varepsilon(y)=\Delta \varepsilon_{1} \cos (K y)+\Delta \varepsilon_{2} \cos (2 K y) \\
+\cdots+\Delta \varepsilon_{n} \cos (n K y)
\end{array}
\end{aligned}
$$

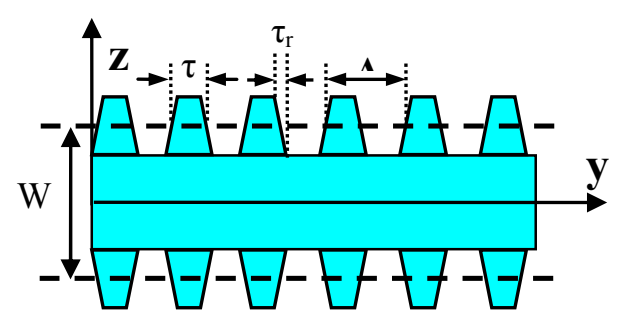

(a)

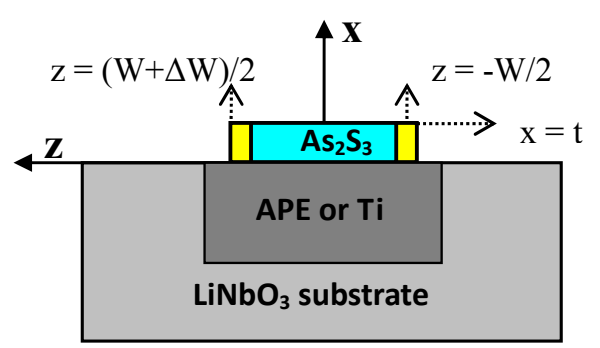

(b)

Figure 2. Top view (a) and cross sectional view (b) of sidewall Bragg gratings on $\mathrm{LiNbO}_{3}$ channel waveguide. $W$ and $t$ are unperturbed $\mathrm{As}_{2} \mathrm{~S}_{3}$ strip width and thickness, respectively. The yellow regions indicate sidewall Bragg gratings. $\Lambda$ and $\Delta W$ are grating period and grating depth, respectively. $\tau$ and $\tau_{r}$ are width of high index and rise width of one period, respectively. 
Here, $\Delta \varepsilon_{n}$ is the Fourier coefficient of the nth harmonic. The influence of each harmonic can be analyzed individually and total effect is obtained by summing all harmonic terms. The values of $\Delta \varepsilon_{n}$ for sinusoidal, square and trapezoidal gratings are given in Equations (6)-(8). Sinusoidal gratings have only the first harmonic. Square and trapezoidal gratings have relatively larger Fourier coefficients of the first order. High order harmonics may be included to achieve the greatest accuracy [10].

Sinusoidal:

$$
\Delta \varepsilon_{1}=\left(\varepsilon_{\mathrm{As}_{2} \mathrm{~S}_{3}}-\varepsilon_{0}\right)
$$

Square:

$$
\Delta \varepsilon_{n}=\frac{4\left(\varepsilon_{\mathrm{As}_{2} \mathrm{~S}_{3}}-\varepsilon_{0}\right)}{n \pi} \sin \left(\frac{n \pi \tau}{\Lambda}\right)
$$

Trapezoidal:

$$
\Delta \varepsilon_{n}=\frac{16\left(\varepsilon_{\mathrm{As}_{2} \mathrm{~S}_{3}}-\varepsilon_{0}\right)}{(n \pi)^{2}} \sin \left(\frac{n \pi \tau}{\Lambda}\right) \sin \left(\frac{n \pi \tau_{r}}{\Lambda}\right)
$$

\subsection{Coupling Coefficients}

The coupling coefficients of sidewall Bragg gratings are evaluated by taking overlap integration between material index distribution and electric field distribution over grating regions as illustrated in Figure 2(b). The coupling coefficient resulting from the nth Fourier harmonic component is computed by

$$
\begin{aligned}
k_{n}=\frac{\omega}{4} \Delta \varepsilon_{n} \cos (n K y) \int_{0}^{t} \mathrm{~d} x & \left\{\int_{(W-\Delta W) / 2}^{(W+\Delta W) / 2} \mathrm{~d} z|E(x, z)|^{2}\right. \\
& \left.+\int_{-(W+\Delta W) / 2}^{-(W-\Delta W) / 2} \mathrm{~d} z|E(x, z)|^{2}\right\}
\end{aligned}
$$

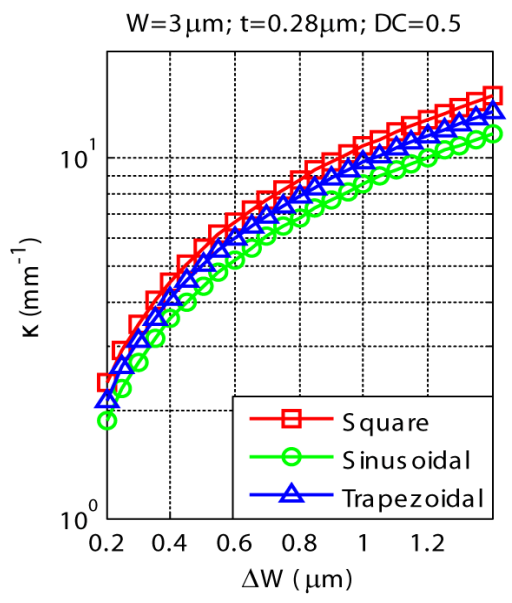

(a) where $\omega=2 \pi c / \lambda_{0}$ is angular frequency; $c$ is vacuum speed of light; $E(x, z)$ is the electric field distribution of $\mathrm{TE}_{0}$ mode supported by the unperturbed $\mathrm{As}_{2} \mathrm{~S}_{3}$ strip.

The coupling coefficients resulting from the first order perturbation for square, sinusoidal and trapezoidal gratings at $\lambda_{0}=1.55 \mu \mathrm{m}$ are displayed in Figure 3. Square gratings posses strongest coupling strength at $k=8.75$ $\mathrm{mm}^{-1}$ due to its largest Fourier coefficient of the first order. As grating depths increases, the coupling coefficient increases. For all the gratings, coupling coefficients are maximized at the duty cycle of 0.5 for a given grating depth.

\subsection{Reflection and Bandwidth}

The reflectance and bandwidth of trapezoidal sidewall gratings with $\Lambda=360 \mathrm{~nm}$ are calculated at $\lambda_{0}=1.55 \mu \mathrm{m}$. Numerical results for weak coupling gratings and strong coupling gratings are plotted in Figures $\mathbf{4}$ and 5, respectively. Under weak coupling, the bandwidth is inversely proportional to grating length $L=N \Lambda$ where $N$ indicates the total number of grating periods. To improve the reflectance of weak coupling gratings and maintain the same grating length, larger grating depth is required. For instance, the grating reflectance for $N=600$ periods can be increased from $60 \%$ to $94 \%$ by varying grating depth from $\Delta W=0.4 \mu \mathrm{m}$ to $\Delta W=1.0 \mu \mathrm{m}$, as shown in Figure 4(a). The sidewall gratings under strong coupling condition have much narrower reflection bandwidth compared with weak coupling gratings. It is also more efficient in reflectance especially at larger values of $\Delta W$. A linear relationship between the bandwidth and the coupling coefficient beyond $k>4 \mathrm{~mm}^{-1}$ is observed in Figure 5(b).

\section{Simulations}

The scattering matrices of the sidewall Bragg gratings

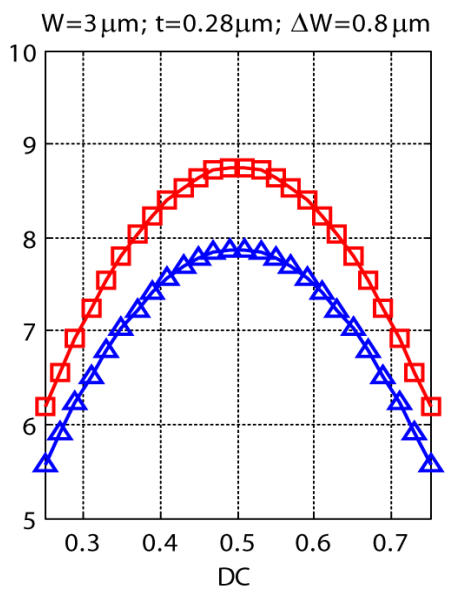

(b)

Figure 3. Effects of perturbation strength $\Delta W$ (a) and duty cycle (b) on coupling coefficients. Red squares, green circles and blue triangles denote square, sinusoidal and trapezoidal gratings, respectively. 


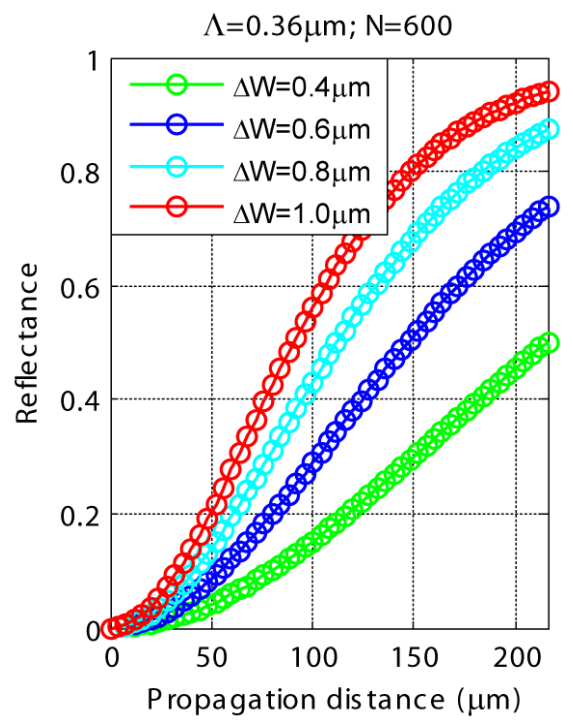

(a)

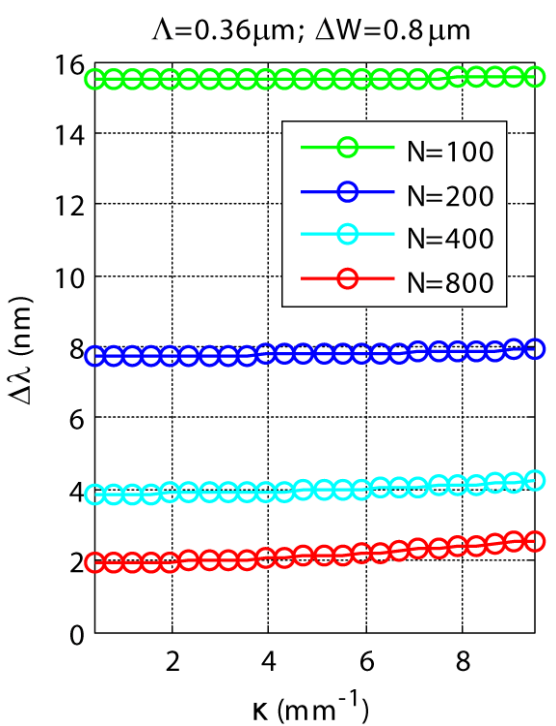

(b)

Figure 4. Reflectance (a) and bandwidth (b) of trapezoidal gratings under weak coupling condition.

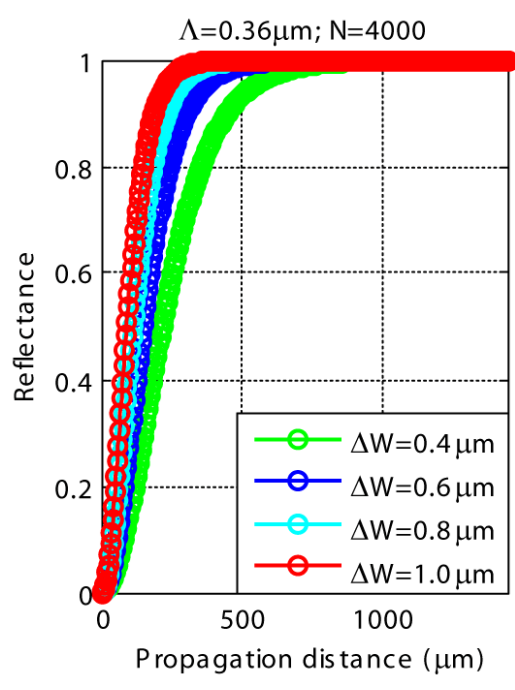

(a)

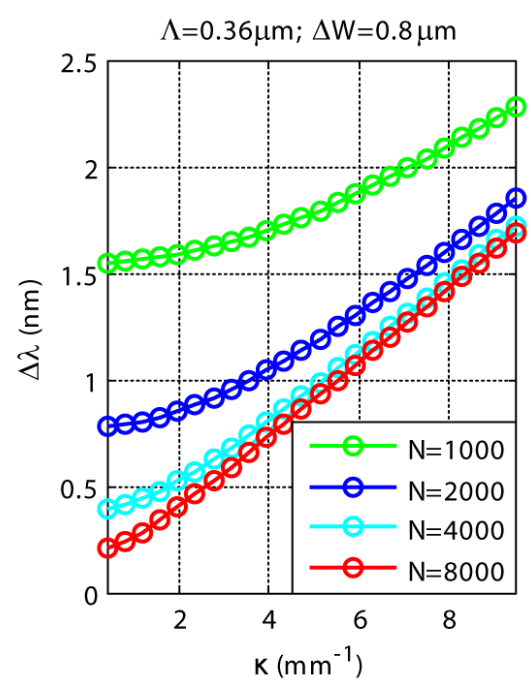

(b)

Figure 5. Reflectance (a) and bandwidth (b) of trapezoidal gratings under strong coupling condition.

are calculated with Fimmprop (Photon Design, Inc., Oxford, UK). The $\mathrm{LiNbO}_{3}$ channel waveguide is fabricated either by APE process or by Ti diffusion process. For $x$-cut $y$-propagation $\mathrm{LiNbO}_{3}$, Ti diffused channel waveguide supports both TE (transverse electric) and TM (transverse magnetic) modes. However, only TE modes are supported by APE channel waveguide because extraordinary refractive index $\left(n_{e}\right)$ of $\mathrm{LiNbO}_{3}$ increases while ordinary refractive index $\left(n_{o}\right)$ decreases during the proton exchanging process [17]. Without any loss in generality, our simulations will focus on TE-polarized sidewall Bragg gratings on APE waveguide. Similar procedure applies for both TE and TM polarizations on Ti diffused $\mathrm{LiNbO}_{3}$ substrate.

\subsection{Uniform Sidewall Gratings}

The thickness of $\mathrm{As}_{2} \mathrm{~S}_{3}$ strip waveguide are optimized at $t$ $=280 \mathrm{~nm}$ for single mode $\mathrm{TE}_{0}$ operation. The effective index of $\mathrm{TE}_{0}$ mode for $W=3 \mu \mathrm{m}$ is $n_{\text {eff }}=2.1436$ at $\lambda_{0}=$ $1.55 \mu \mathrm{m}$. The Bragg grating period is $\Lambda=\lambda_{0} /\left(2 n_{\text {eff }}\right)=$ $361.5 \mathrm{~nm}$. We choose $\Lambda=360 \mathrm{~nm}$ for easy fabrication. Linear tapers with a tip width of $350 \mathrm{~nm}$ and length of $500 \mu \mathrm{m}$ is also designed to transfer optical mode power between APE channel waveguide and $\mathrm{As}_{2} \mathrm{~S}_{3}$ strip waveguide.

The reflectance of trapezoidal Bragg gratings with DC $=0.5$ and $\tau_{r} / \Lambda=0.25$ under weak coupling and strong coupling condition is shown in Figures 6-8. Generally, 


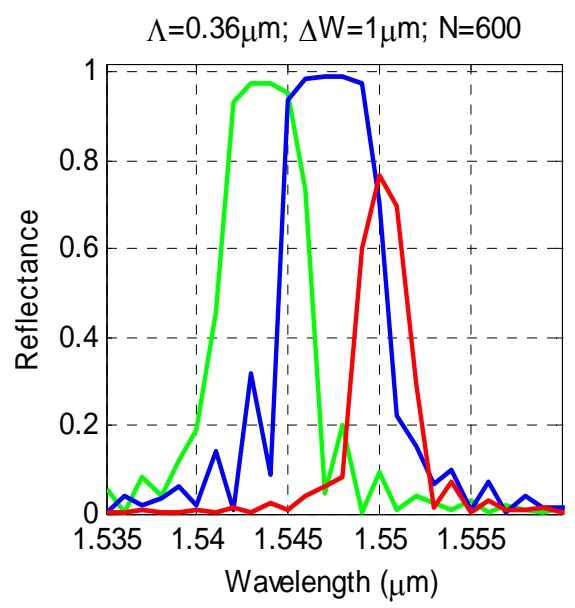

(a)

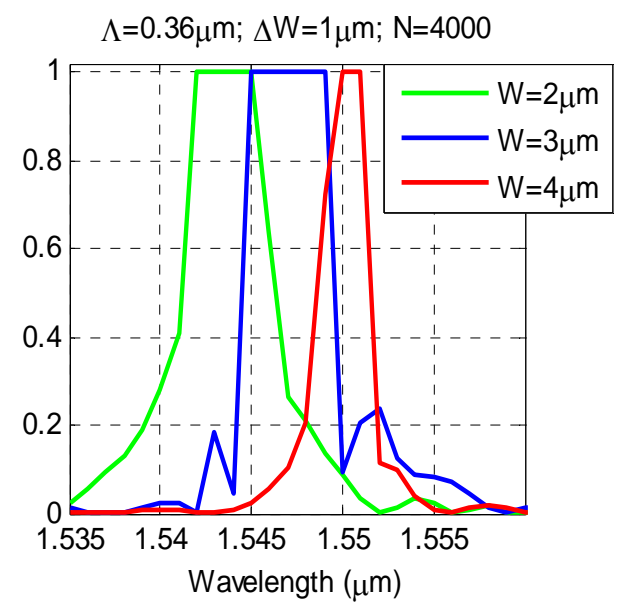

(b)

Figure 6. Reflectance of trapezoidal sidewall gratings nder weak coupling (a) and strong coupling condition (b) for different unperturbed $\mathrm{As}_{2} \mathrm{~S}_{3}$ strip widths.

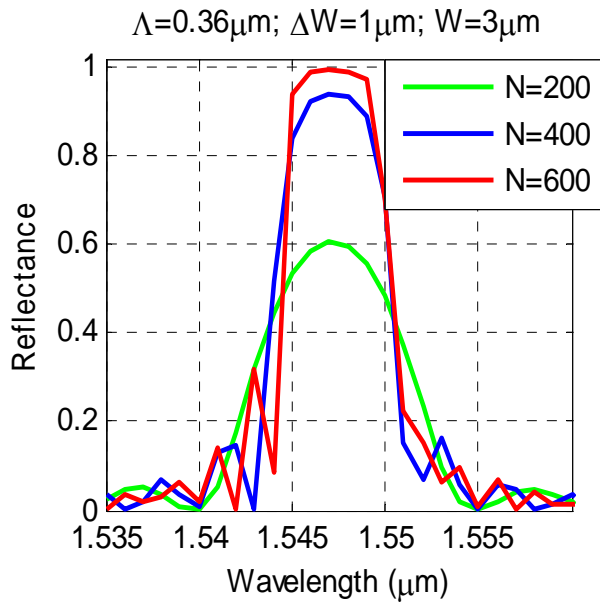

(a)

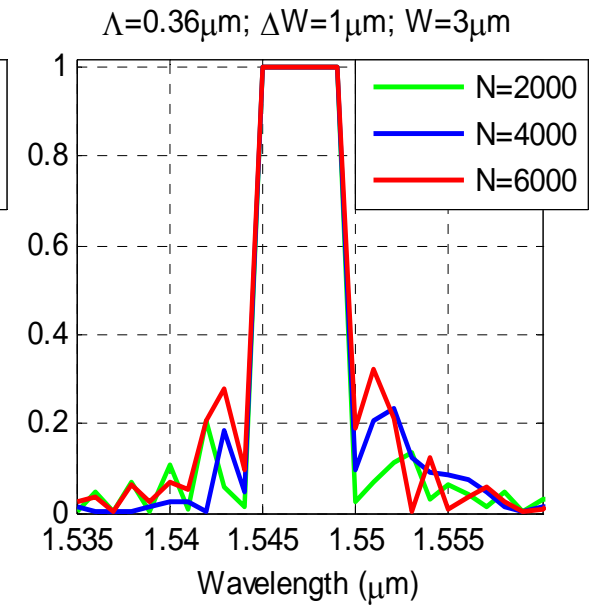

(b)

Figure 7. Reflectance of trapezoidal sidewall gratings nder weak coupling (a) and strong coupling condition (b) for different numbers of grating periods.

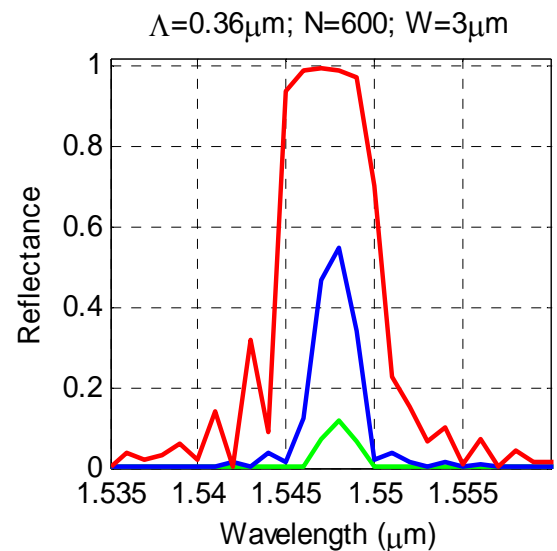

(a)

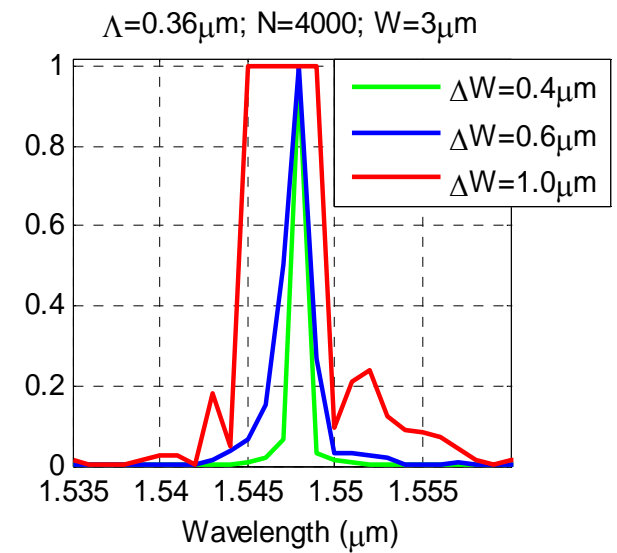

(b)

Figure 8. Reflectance of trapezoidal sidewall gratings under weak coupling (a) and strong coupling condition (b) for different grating depths. 
the reflectance spectra of weak coupling gratings exhibits the shape of sinc function while the reflectance of strong coupling gratings have a flat top in the reflection band. Because the effective index is larger for a wider $\mathrm{As}_{2} \mathrm{~S}_{3}$ strip, the Bragg wavelength is proportional to the effective mode index for a given grating period according to Equation (6). So the Bragg wavelength can be located to a desired value by varying the unperturbed $\mathrm{As}_{2} \mathrm{~S}_{3}$ strip width as shown in Figure 6. The side-lobe levels can be suppressed by anodizing these sidewall gratings in order to minimize cross talk between channels and improve filter responses for add/drop filters used in dense wavelength division multiplexing (DWDM) [11].

For weak coupling gratings, a reflection band of $\sim 5 \mathrm{~nm}$ centered at $\sim 1.548 \mu \mathrm{m}$ (corresponding to $\Lambda=360 \mathrm{~nm}$ ) is observed in Figure 7(a) for $N=600$ periods. The reflectance increases with increasing numbers of grating periods, however, the reflection bandwidth gets narrower. As shown in Figure 8(a), the grating depth needs to be sufficiently large to achieve a high reflectance for weak coupling sidewall gratings. Under strong coupling condition, the reflectance is very efficient and the bandwidth is determined only by coupling coefficients, as shown in Figures 7(b) and 8(b).

\subsection{Phase-Shifted Sidewall Gratings}

The uniform sidewall grating with $N=600$ periods can be spitted into halves by inserting a phase-shifting spacer with the length $L_{s}=\Lambda / 2$ (i.e., the quarter-wavelength length $\left.\lambda /\left(4 n_{\text {eff }}\right)\right)$. As a result, a Fabry-Perot type of optical resonant cavity with a length of $L_{s}$ is formed by two identical distributed Bragg reflectors (DBRs) [28]. A transmission peak will be created at the center of the stopband of the uniform sidewall gratings [7,9]. As shown in Figure 9(a), the red line indicates the reflectance spectrum from a uniform trapezoidal sidewall grating with $N=600, W=$ $3 \mu \mathrm{m}, \Delta W=1.0 \mu \mathrm{m}$ and $\mathrm{DC}=0.5$ and the blue line denotes the reflectance from a phase-shifted sidewall grating. A transmission band centered at $1.5472 \mu \mathrm{m}$ with a bandwidth of $0.45 \mathrm{~nm}$ is observed in the center of $\Delta \lambda=$ $12 \mathrm{~nm}$ stopband of the $N=600$ uniform sidewall gratings. The quality factor (Q-factor) of this resonant cavity is $\mathrm{Q}$ $=\lambda_{0} / \Delta \lambda=3.4 \times 10^{3}$ which has the same order as that of the transmission resonant filter in SOI (silicon on insulator) material system in Ref. [9].

Besides, multi-channel transmission filters can be implemented by introducing multiple phase-shifting spacers in uniform sidewall gratings [6,12]. For instance, Figure 9(b) displays the reflectance spectra from sidewall gratings with single, double and triple phase shifting spacers, where each grating section has $N / 2=300$ periods and each phase shifting spacer has the quarter-wavelength length. Add/drop filters for DWDM in telecommunication can be implemented with such phase shifted sidewall gratings [6,28].

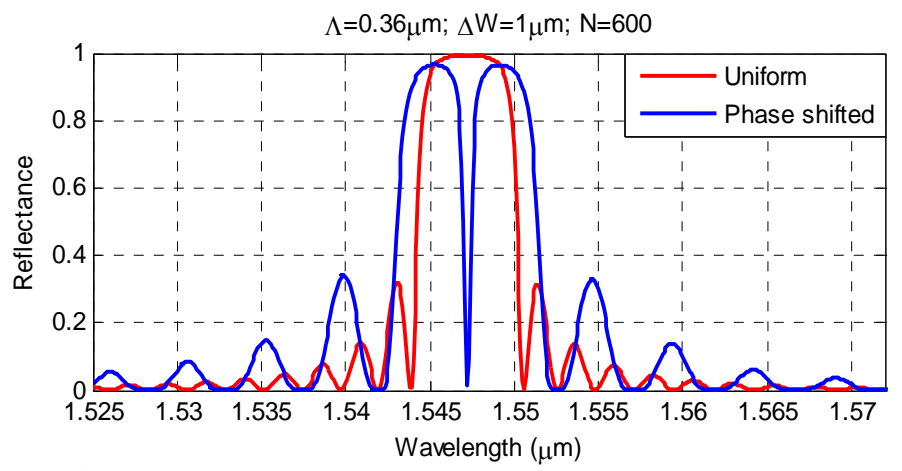

(a)

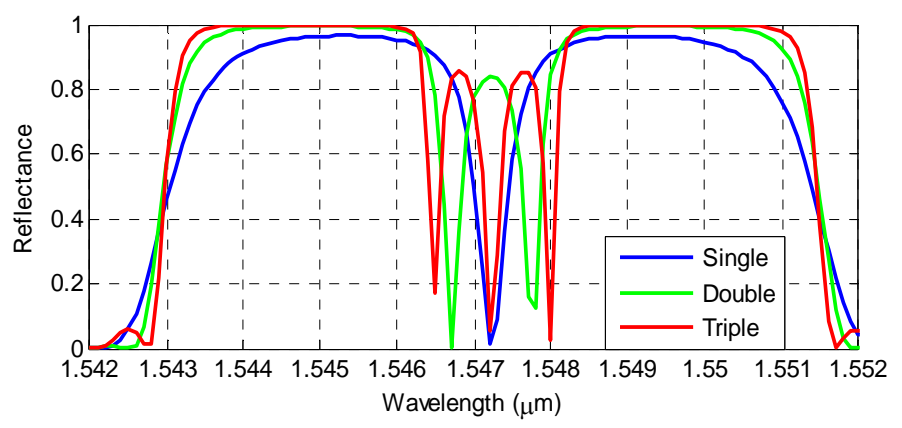

(b)

Figure 9. Reflectance from uniform sidewall gratings and phase-shifted sidewall Bragg gratings. 


\subsection{Sidewall Gratings Resonant Cavities}

Similar to the phase-shifted sidewall gratings, an optical resonant cavity with the length of $L_{c}$ can be constructed by two identical DBRs. This optical resonant cavity can be treated as a Fabry-Perot resonator with an effective cavity length $L_{\text {eff }}$ [24]. The reflectance spectra for three optical resonant cavities with length $L_{c}=0.3 \mathrm{~mm}, L_{c}=$ $0.5 \mathrm{~mm}$ and $L_{c}=0.7 \mathrm{~mm}$ are given in Figure 10(a). The free spectral range (FSR) is inversely proportional to the effective cavity length and is determined by Equation (10). For the cavity with $L_{c}=0.5 \mathrm{~mm}$, the resonant transmission bandwidth is $0.04 \mathrm{~nm}$ at $1.5496 \mu \mathrm{m}$ and thus the Q-factor is $\sim 3.874 \times 10^{4}$. The FSR is $0.9 \mathrm{~nm}$ $(\sim 112 \mathrm{GHz})$. The effective cavity length is estimated at $L_{\text {eff }}=0.62 \mathrm{~m}$ which is larger than its physical length. The FSR and Q-factor for the $L_{c}=0.7 \mathrm{~mm}$ cavity are $0.63 \mathrm{~nm}$ and $9.68 \times 10^{4}$, respective. It is expected that resonant cavities with a Q-factor over $10^{6}$ can be realized with the physical cavity length longer than $1 \mathrm{~cm}$. The spectral transmission of one ultrahigh Q-factor resonant cavity with the cavity length of $1.2 \mathrm{~cm}$ is plotted in Figure 10(b). It demonstrates the FSR (and Q-factor of this resonant cavity are $42.5 \mathrm{pm}$ and $1.21 \times 10^{6}$. Therefore, such sidewall Bragg gratings are very useful for compact resonant cavities with ultrahigh Q-factors at both near-IR and mid-IR wavelengths.

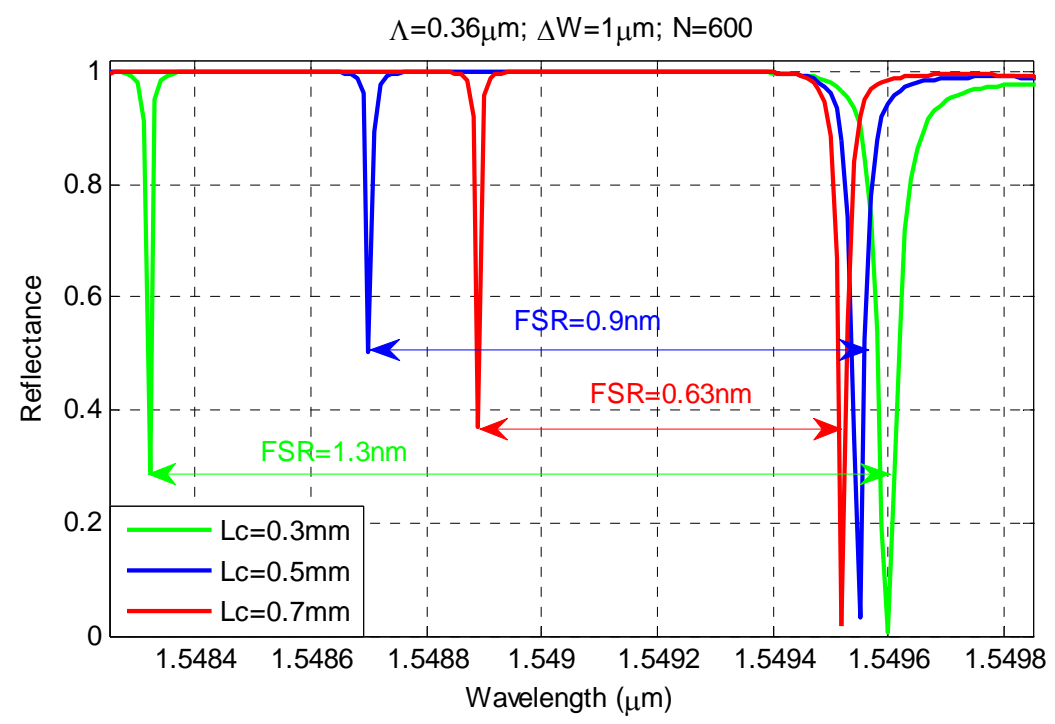

(a)

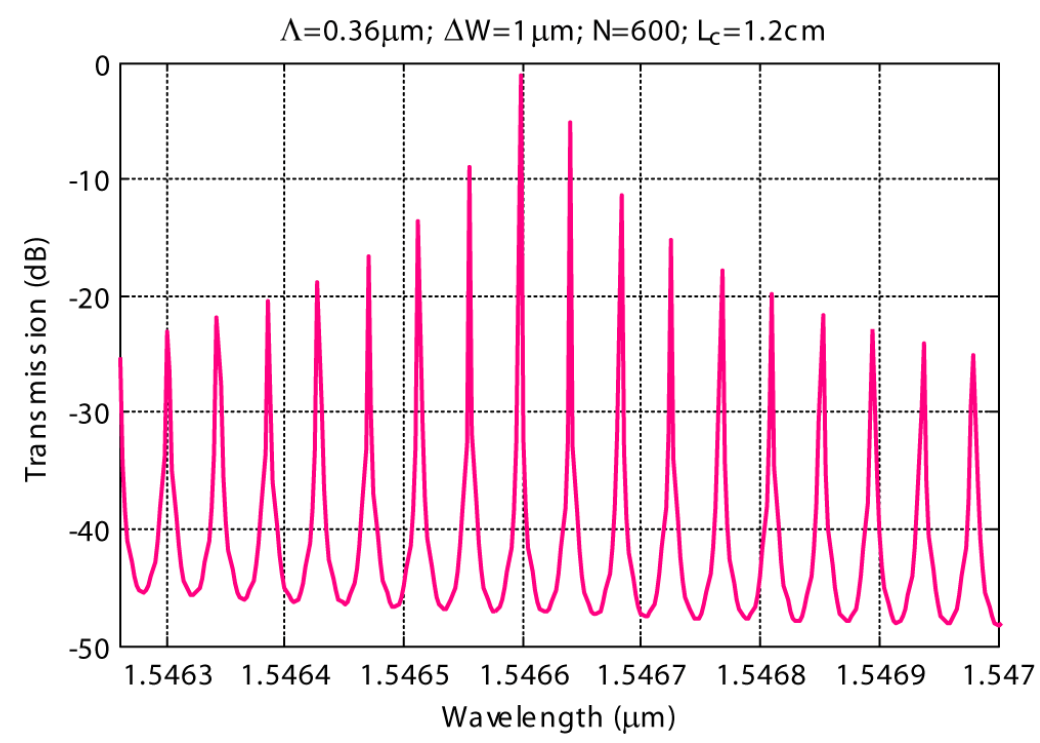

(b)

Figure 10. Reflectance of resonant cavities formed by sidewall Bragg gratings with different cavity lengths (a). Transmittance of ultrahigh Q-factor resonant cavities with $L_{c}=1.2 \mathrm{~cm}(\mathrm{~b})$. 


$$
F S R=\frac{\lambda^{2}}{2 n_{\mathrm{eff}} L_{\mathrm{eff}}}
$$

\subsection{Electro-Optic Tunable Sidewall Gratings}

Electro-optic tunable Bragg gratings engraved on Ti diffused waveguide was reported with tuning efficiency at $\sim 5 \mathrm{pm} / \mathrm{V}$ [29]. Here we will investigate the electro-optical tenability of sidewall Bragg gratings on APE waveguides. The electro-optic (EO) effect, or Pockels effect, is exhibited by non-centrosymmetric crystals such as lithium niobate [15]. For the $\mathrm{As}_{2} \mathrm{~S}_{3}$ sidewall gratings on APE $\mathrm{LiNbO}_{3}$ channel waveguide shown in Figure 11, optical mode is partially confined in $\mathrm{As}_{2} \mathrm{~S}_{3}$ strip ( $\sim 38 \%$ at $1.55 \mu \mathrm{m}$ for $W=3 \mu \mathrm{m}$ and $t=280 \mathrm{~nm})$ and the rest of mode is confined in APE channel waveguide, as shown in Figure 11(b). This hybrid mode feature along with EO property of $\mathrm{LiNbO}_{3}$ substrate facilitates electro-optic tuning of such sidewall gratings. On the $x$-cut $y$-propagation $\mathrm{LiNbO}_{3}$ substrate, the optical electric field along the $z$-axis will experience optimal EO effect due to its highest value of EO coefficient $r_{33}=30.8 \times 10^{-12} \mathrm{~m} / \mathrm{V}$. As illustrated in Figure 11(a), if the gap between the two parallel electrodes is $d$, the electric field from external applied voltage $V_{a}$ along $z$-axis is given by

$$
E_{z}=\frac{V_{a}}{d} \text {. }
$$

The corresponding amount of refractive index change along $z$-axis (i.e., extraordinary refractive index) is determined by

$$
\Delta n_{e}=-\frac{1}{2} n_{e}^{3} r_{33} E_{z} .
$$

Assume $d=10 \mu \mathrm{m}$ and $V_{a}=50 \mathrm{~V}$, then $\Delta n_{e}=-8.199$ $\times 10^{-4}$. Figure 11(c) gives electric field distribution generated from the external applied voltage at $50 \mathrm{~V}$. This electric field induces an amount of refractive index change of $-4.8579 \times 10^{-4}$, which is smaller than the theoretical value because only up to $75 \%$ of the bulk value of $r_{33}$ can be restored by annealing process after proton exchange [30]. The reflectance of the single phase-shifted sidewall grating in Section 3.2 with different external applied voltages is plotted in Figure 12. EO tuning efficiency at $\sim 4 \mathrm{pm} / \mathrm{V}$ is achieved.

\section{Conclusions}

In summary, the spectral properties of $\mathrm{As}_{2} \mathrm{~S}_{3}$ sidewall gratings integrated on $\mathrm{LiNbO}_{3}$ substrate were analyzed with coupled-mode theory. Coupling coefficients were evaluated by performing overlap integration. Numerical results of uniform sidewall gratings agree well with the coupled-mode theory. Single and multi-channel transmission resonant filters based on sidewall Bragg gratings

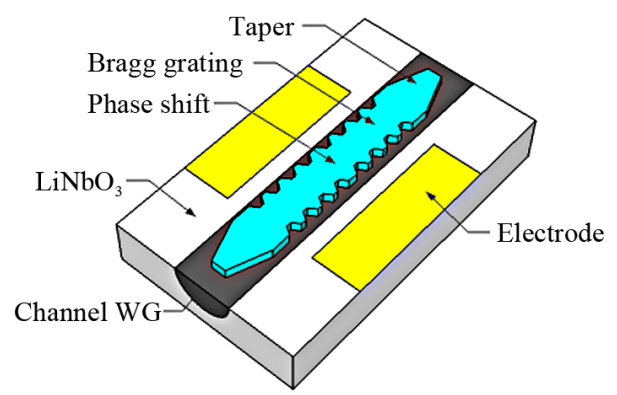

(a)

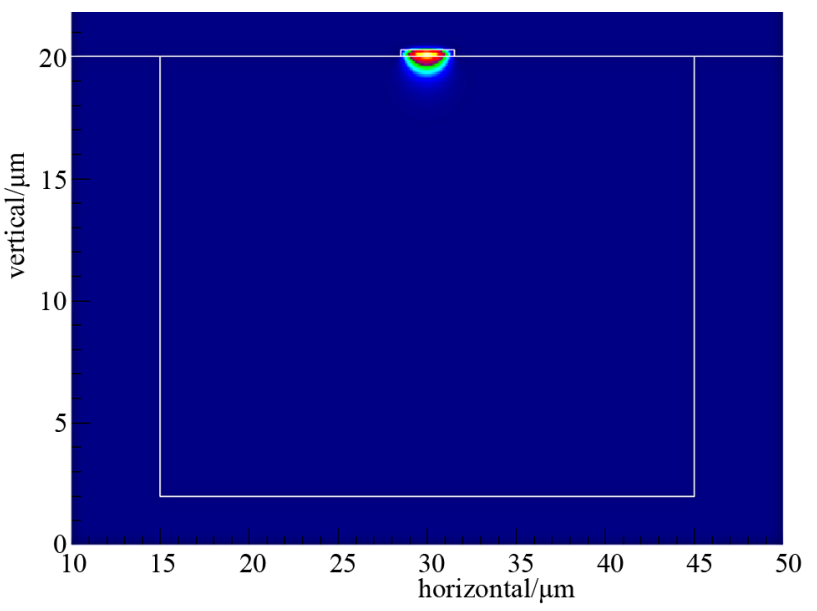

(b)

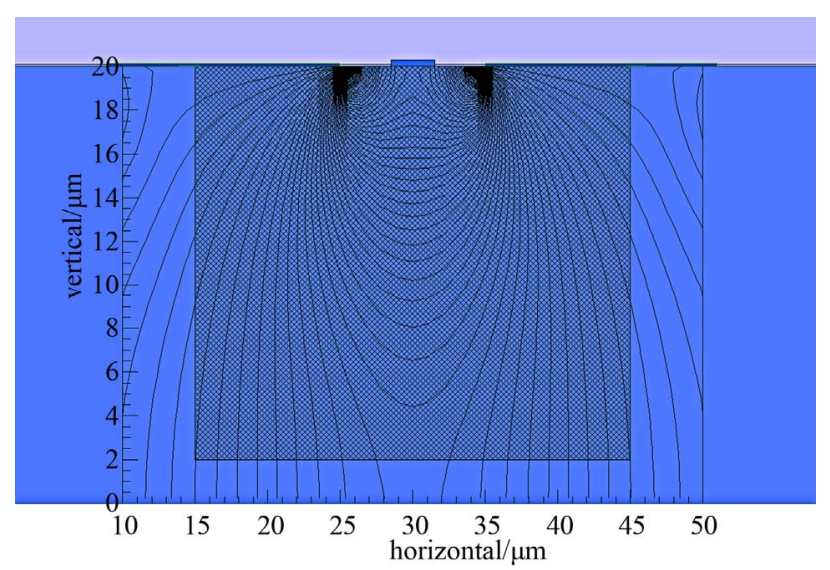

(c)

Figure 11. Simulation model for the EO tunable phaseshifted transmission resonant filter (a). The optical intensity of the electrical field component along $z$-axis of $\mathrm{TE}_{0}$ mode (b) and the electric field profile with externally applied voltage at $V_{a}=50 \mathrm{~V}$ (c).

were discussed. An electro-optic tunable narrowband transmission resonant filter was proposed and a tuning rate of $\sim 4 \mathrm{pm} / \mathrm{V}$ was realized. Lithium niobate channel waveguides with low propagation loss can be prepared by thin film Ti diffusion as well as annealed proton exchange process. Such $\mathrm{As}_{2} \mathrm{~S}_{3}$ sidewall gratings with nanoscale grating periods can be easily fabricated by direct electron beam writing or focused ion beam lithography. 


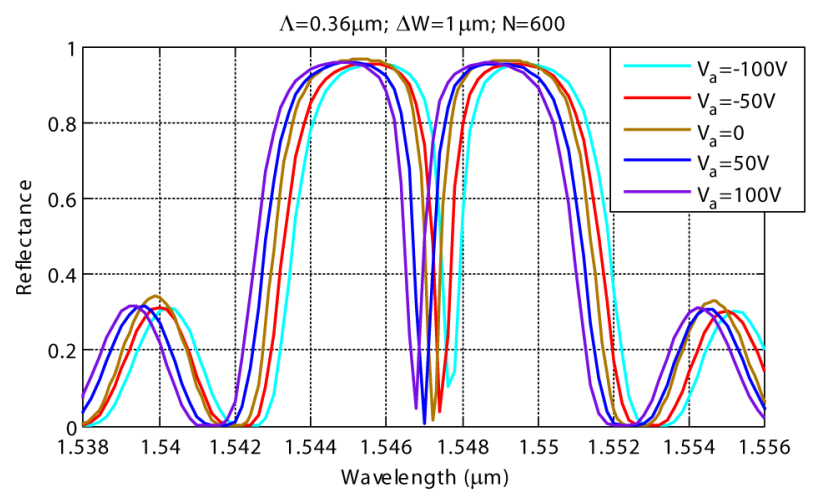

Figure 12. Reflectance of EO tunable sidewall gratings at different externally applied voltages.

This type of integrated chacogenide $\mathrm{As}_{2} \mathrm{~S}_{3}$ sidewall Bragg gratings integrated on $\mathrm{LiNbO}_{3}$ substrate provide an approach for the design of a wide range of integrated-optic devices such as switches, laser cavities, modulators, sensors and tunable filters.

\section{REFERENCES}

[1] A. Melloni, M. Chinello and M. Martinelli, "All-Optical Switching In Phase-Shifted Fiber Bragg Grating," IEEE Photonics Technology Letters, Vol. 12, No. 1, 2000, pp. 42-44. doi:10.1109/68.817464

[2] L. Pierno, M. Dispenza, A. Secchi, A. Fiorello and V. Foglietti, "A Lithium Niobate Electro-Optic Tunable Bragg Filter Fabricated by Electron Beam Lithography," Journal of Optics A: Pure and Applied Optics, Vol. 10, No. 6, 2008, Article ID: 064017. doi:10.1088/1464-4258/10/6/064017

[3] B. K. Das, R. Richen and W. Sohler, "Integrated Optical Distributed Feedback Laser with Ti:Fe:Er:LiNbO 3 Waveguide," Applied Physics Letters, Vol. 82, No. 10, 2003, pp. 1515-1517. doi:10.1063/1.1559443

[4] R. Kim, J. Zhang, O. Eknoyan, H. F. Taylor and T. L. Smitch, "Fabry-Perot Intensity Modulator with Integrated Bragg Reflectors in Ti:LiNbO 3 ," Electronics Letters, Vol. 41, 2005, pp. 1220-1222. doi:10.1049/el:20053038

[5] J. H. Song, J. H. Lim, R. K. Kim, K. S. Lee, K.-Y. Kim, J. Cho, D. Han, S. Jung, Y. Oh and D.-H. Jang, "Bragg Grating-Assisted WDM Filter for Integrated Optical Triplexer Transceivers," IEEE Photonics Technology Letters, Vol. 17, No. 12, 2005, pp. 2607-2609.

doi:10.1109/LPT.2005.859181

[6] L. Zhu, Y. Huang, W. M. J. Green and A. Yariv, "Polymeric Multi-Channel Bandpass Filters in Phase-Shifted Bragg Waveguide Gratings by Direct Electron Beam Writing," Optics Express, Vol. 12, No. 25, 2004, pp. 6372-6376. doi:10.1364/OPEX.12.006372

[7] H.-C. Kim, K. Ikeda and Y. Fainman, "Tunable Transmission Resonant Filter and Modulator with Vertical Gratings," Journal of Lightwave Technology, Vol. 25, No. 5, 2007, pp. 1147-1151. doi:10.1109/JLT.2007.893922

[8] Y.-B. Cho, B.-K. Yang, J.-H. Lee and J.-B. Yoon, "Sili- con Photonic Wire Filter Using Asymmetric Sidewall Long-Period Waveguide Grating in a Two-Mode Waveguide," IEEE Photonics Technology Letters, Vol. 20, No. 7, 2008, pp. 520-522. doi:10.1109/LPT.2008.918895

[9] H.-C. Kim, K. Ikeda and Y. Fainman, "Resonant Waveguide Device with Vertical Gratings," Optics Letters, Vol. 32, No. 5, 2007, pp. 539-541. doi:10.1364/OL.32.000539

[10] R. Millett, K. Hinzer, A. Benhsaien, T. J. Hall and H. Schriemer, "The Impact of Laterally Coupled Grating Microstructure on Effective Coupling Coefficients," Nanotechnology, Vol. 21, No. 13, 2010, Article ID: 134015. doi:10.1088/0957-4484/21/13/134015

[11] J. T. Hastings, M. H. Lim, J. G. Goodberlet and H. I. Smith, "Optical Waveguides with Apodized Sidewall Gratings via Spatial-Phase-Locked Electron-Beam Lithography," Journal of Vacuum Science \& Technology B, Vol. 20, No. 6, 2002, pp. 2753-2757. doi:10.1116/1.1521744

[12] A. Lupu, K. Muhieddine, E. Cassan and J.-M. Lourtioz, "Dual Transmission Band Bragg Grating Assisted Asymmetric Directional Couplers," Optics Express, Vol. 19, No. 2, 2011, pp. 1246-1259. doi:10.1364/OE.19.001246

[13] P. Ma, Y. Fedoryshyn and H. Jachel, "Ultrafast All-Optical Switching Based on Cross Modulation Utilizing Intersubband Transitions in InGaAs/AlAs/AlAsSb Coupled Quantum Wells with DFB Grating Wave- guides," Optics Express, Vol. 19, No. 10, 2011, pp. 9461-9474. doi:10.1364/OE.19.009461

[14] P. Prahbathan, V. M. Murukeshan, Z. Jing and P. V. Ramana, "Compact SOI Nanowire Refractive Index Sensor Using Phase Shifted Bragg Gratings," Optics Express, Vol. 17, No. 17, 2009, pp. 15330-15341. doi:10.1364/OE.17.015330

[15] A. M. Prokhorov, Y. S. Kuzminov and O. A. Khachaturyan, "Ferroelectric Thin-Film Waveguides in Integrated Optics and Optoelectronics," Cambridge International Science Publishing, Cambridge, 1997.

[16] B.-E. Benkelfat, T. Ferriere, B. Wacogne and P. Mollier, "Technological Implementation of Bragg Gratings Reflectors in Ti: $\mathrm{LiNbO}_{3}$ Waveguides by Proton Exchange," IEEE Photonics Technology Letters, Vol. 14, No. 10, 2002, pp. 1430-1432. doi:10.1109/LPT.2002.801106

[17] C. Madsen, W. C. Tan, X. Xia, W. Snider and I. Zhou, "Hybrid Chalcogenide/Lithium Niobate Waveguides," Conference on Novel Hybrid Integration, Frontiers in Optics (FiO) 2010/Laser Science (LS) XXVI, Rochester, 24-28 October 2010.

[18] Y. Ruan, W. Li, R. Jarvis, N. Madsen, A. Rode and Luther-Davis, "Fabrication and Characterization of Low Loss Rib Chalcogenide Waveguides Made by Dry Etching," Optics Express, Vol. 12, No. 21, 2004, pp. 51405145. doi:10.1364/OPEX.12.005140

[19] X. Xia, Q. Chen, C. Tsay, C. B. Arnold and C. K. Madsen, "Low-Loss Chalcogenide Waveguides on Lithium Niobate for the Mid-Infrared," Optics Letters, Vol. 35, No. 19, 2010, pp. 3228-3230. doi:10.1364/OL.35.003228

[20] C. Madsen, M. Solmaz and R. Atkins, "High-Index-Contrast Chalcogenide Waveguides," Proceedings of SPIE, Vol. 6897, 2008, Article ID: 689703. 
doi: $10.1117 / 12.768583$

[21] M. E. Solmaz, D. B. Adams, S. Grover, W.-C. Tan, X. Xia, O. Eknoyan and C. K. Madsen, "Compact Bends for Achieving Higher Integration Densities for $\mathrm{LiNbO}_{3}$ Waveguides," IEEE Photonics Technology Letters, Vol. 21, No. 9, 2009, pp. 557-559. doi:10.1109/LPT.2009.2014569

[22] Y. Zhou, X. Xia, W. T. Snider, J. Kim, Q. Chen, W. C. Tan and C. K. Madsen, "Two-Stage Taper Enhanced Ultra-High Q $\mathrm{As}_{2} \mathrm{~S}_{3}$ Ring Resonator on $\mathrm{LiNbO}_{3}$," IEEE Photonics Technology Letters, Vol. 23, 2011, pp. 11951197.

[23] D. Lee, "Electromagnetic Principles of Integrated Optics," Jphn Wiley \& Sons, New York, 1986, Chap. 8.

[24] A. Yariv and A. P. Yeh, "Photonics: Optical Electronics in Modern Communications," 6th Edition, Oxford University Press, New York, 2007.

[25] J. Hong and W. Huang, "Contra-Directional Coupling in Grating-Assisted Guided-Wave Devices," Journal of Lightwave Technology, Vol. 10, No. 7, 1992, pp. 873-881. doi:10.1109/50.144907

[26] U. Schlarb and K. Berzler, "A Generalized Sellmeier Equation for the Refractive Indices of Lithium Niobate,"
Ferroelectrics, Vol. 156, No. 1, 1993, pp. 99-104. doi:10.1080/00150199408215934

[27] C. Chaudhari, T. Suzuki and Y. Ohishi, "Design of Zero Chromatic Dispersion Chalcogenide $\mathrm{As}_{2} \mathrm{~S}_{3}$ Glass Nanofibers," Journal of Lightwave Technology, Vol. 27, No. 12, 2009, pp. 2095-2099. doi:10.1109/JLT.2008.2007223

[28] P. Prabhathan, V. M. Murukeshan and Z. Jing, "Compact Resonant Bragg Grating Filters Using Submicron Silicon-on-Insulator (SOI) Waveguide for Optical Communication Network," Proceedings of SPIE, Vol. 7847, 2010, Article ID: 74870.

[29] L. Pierno, M. Dispenza, A. Secchi, A. Fiorello and V. Foglietti, "A Lithium Niobate Electro-Optic Tunable Bragg Filter Fabricated by Electron Beam Lithography," Journal of Optics A: Pure and Applied Optics, Vol. 10, No. 6, 2008, Article ID: 064017. doi:10.1088/1464-4258/10/6/064017

[30] I. Savatinova, S. Tonchev, R. Todorov, M. N. Armenise, V. M. N. Passaro and C. C. Ziling, "Electro-Optic Effect in Proton Exchanged $\mathrm{LiNbO}_{3}$ and $\mathrm{LiTaO}_{3}$ Waveguides," Journal of Lightwave Technology, Vol. 14, No. 3, 1996, pp. 403-409. doi:10.1109/50.485600 